\title{
ON ADHESIVE THEORIES IN MULTILAYERED INTERFACES, WITH PARTICULAR REGARD TO "SURFACE FORCE APPARATUS" GEOMETRY
}

\author{
Michele Tricarico ${ }^{1}$, Antonio Papangelo ${ }^{2}$, \\ Andrei Constantinescu ${ }^{3}$, Michele Ciavarella ${ }^{1}$
}

${ }^{1}$ Politecnico di Bari, Department of Mechanics, Mathematics and Management, Italy ${ }^{2}$ Hamburg University of Technology, Department of Mechanical Engineering, Germany ${ }^{3}$ Laboratoire de Mécanique des Solides, Ecole Polytechnique, Palaiseau cedex, France

\begin{abstract}
Adhesion is a key factor in many tribological processes, especially wear. We generalize a recent formulation for the indentation of a multilayered material using an efficient integral transform method, to the case of adhesion, using a simple energetic transformation in the JKR regime. Then, we specialize the study for the geometry of the Surface Force Apparatus, which consists of two thin layers on a substrate, where the intermediate layer is softer than the other two. We find the pull-off force under "force control" (i.e. for "soft" loading systems), as well as under "displacement control" (i.e. for "rigid" systems), as a function of the geometrical thicknesses and material properties ratios, and the method is fully implemented in a fast Mathematica code, available to the public (see Appendix).
\end{abstract}

Key Words: JKR Theory, Surface Force Apparatus, Adhesion

\section{INTRODUCTION}

Adhesion forces are more and more of interest in many areas of engineering, both as the basic building block to the theories of frictional interaction, or wear, particularly at micro and nano scales, i.e. at the level of asperities. Wear in particular does not occur when asperities deform plastically but when they adhere to the countersurface so strongly that they in fact detach a particle of material.

Received January 18, 2019 / Accepted March 08, 2019

Corresponding author: Michele Ciavarella

Department of Mechanics, Mathematics and Management, Politecnico di Bari, Viale Japigia, Italy

E-mail:mciava@poliba.it 
In a recent paper [1], the role of adhesion in contact mechanics has been reviewed starting in particular from the fundamental contribution of Johnson, Kendall and Roberts (JKR) [2], who generalized Hertz' theory to include van der Waals forces described as infinitely short range forces, so that a "contact area" can still be defined, which includes both compressive and tensile stresses. JKR theory has been confirmed in a number of investigations: in principle it should hold only for soft materials and large sphere radius, but in practice for a single, smooth asperity, it holds approximately even for dimensions appropriate to the Atomic Force Microscope (AFM), or the Surface Force Apparatus (SFA). The latter is a scientific instrument which measures the interaction force of two surfaces as they are brought together and retracted using multiple beam interferometry to monitor surface separation, directly measure the contact area and observe any surface deformations occurring in the contact zone. Developed by Tabor and Winterton [3] and Israelachvili and Tabor [4], it comprises thin sheets of molecularly smooth mica, or similar material, glued to the cylindrical glass lenses of equal radii, which are then pressed into elastic contact with their axes at right angles. The SFA is frequently used in conjunction with the JKR theory to extract the surface energy of the contacting sheets. Errors may arise since the JKR theory accounts for the contact of homogeneous, isotropic and elastic cylinders (equivalent to the contact of a sphere with a flat surface). Sridhar et al. [5] extended the JKR theory to the layered structure of the SFA.

The main condition for applying the JKR is perhaps that surfaces should be very smooth, and this is why the surface layer of the SFA is an extremely flat, optically transparent, mica layer (in turn backed with an ultra-thin silver layer to reflect light), and further material or molecules of interest are then coated or adsorbed onto the mica layer. The mica layer is mounted on a glass cylinder and two such cylinders are put in contact in cross-perpendicular configuration, which is equivalent to the contact of a sphere with a plane (see Fig.1). SFA is extremely sensitive as it uses piezoelectrics to position with a force accuracy in forces at the $10^{-8} \mathrm{~N}$ level, and optical interferometry to measure distances to within 0.1 nanometer, and is similar in some respects to the Atomic Force Microscope (AFM), except that it is a surfacesurface apparatus rather than a tip-surface one, and it can measure much longer-range forces, although as a technique it is probably more laborious.

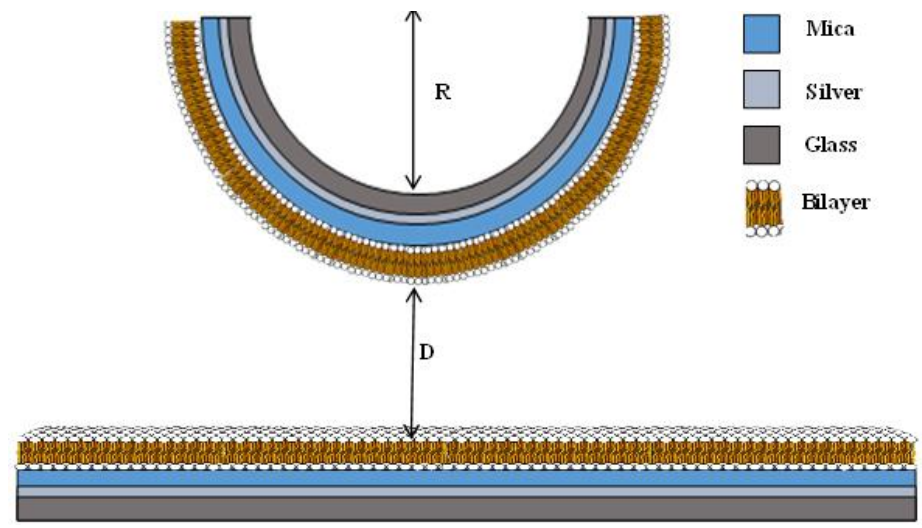

Fig. 1 The geometry of the SFA apparatus contact. In each of the crossing cylinders, a mica layer is covering a glass cylinder (a bilayer is in this configuration adsorbed) 
In the SFA, many authors probably use the JKR original formulation for homogeneous halfspaces to extract the surface work of adhesion of the contacting sheets. Sridhar et al. [5] already proposed a hybrid Finite Element Method-analytical technique to obtain an extension of the JKR solution. Their work plots the force as a function of the contact area, and hence reveals only the maximum force under force control (when the loading system can be considered "soft"), which they find only weakly dependent on moduli or thicknesses ratios.

As an example, we can consider the case of Functionally Graded Materials with power law elastic modulus, where $E_{0}$ is the characteristic modulus at length $z_{0}$

$$
E(z)=E_{0}\left(\frac{z}{z_{0}}\right)^{k} \text { with }-1 \leq k \leq 1
$$

This case can be solved analytically [6], to obtain that the pull-off force remains independent on the elastic modulus

$$
P_{c}=-\frac{k+3}{2} \pi R \varpi
$$

[for example under force control] where $\omega$ is the work of adhesion of the interface. Hence, $P_{c}$ is very weakly dependent on power exponent $k$ and for example for a case $k=0.5$ the pull-off changes from the $J K R$ value $P_{c}=-\frac{3}{2} \pi R \varpi$ by only $+14 \%$.

However, a combination of elastic moduli which is not monotonically varying leads to more interesting results. For example, Stan and Adams [7] use a mathematical solution similar to what we shall use here, and show the results for three samples having layer structure (from top to bottom) indicated in Tab. 1. The samples differ from each other through the elastic modulus of the second layer where sample \#3 is very soft. Each layer was $2 \mathrm{~nm}$ thick, the tip was considered rigid and of radius $20 \mathrm{~nm}$, and $\mathrm{w}=0.1 \mathrm{~J} / \mathrm{m}^{2}$ for all the samples. Sample \#1, \#2 give almost identical results, while sample \#3 gives a very different force-indentation curve, with less pull-off under force control, but much more pull-off under displacement control, perhaps by $+30-40 \%$, than the other two.

Table 1 Examples of 3 layers structure on a substrate of much larger modulus (100 GPa, Poisson's ratio 0.25), in Stan and Adams [7]

\begin{tabular}{cccc}
\hline$E[\mathrm{GPa}]$ & $\# 1$ & $\# 2$ & $\# 3$ \\
\hline Top & 15 & 15 & 15 \\
Mid & 50 & 25 & 5 \\
Bott & 10 & 10 & 10 \\
\hline
\end{tabular}

McGuiggan et al.[8] used the FEM technique of [5] to find more extensive results for the SFA including some experiments, and found that, realistically, the pull-off force can vary between

$$
P_{c}=-\pi R \varpi \ldots-2 \pi R \varpi
$$


i.e. a variation with respect to the JKR value of $-33 \% \ldots+33 \%$. Material properties and layer thicknesses in [8] are reported in Tab. 2, and can be considered typical for SFA. Between the glass substrate (silica) and the mica surface, which are almost of the same material properties, there is an intermediate layer with more than one order of magnitude softer modulus, which is typically an epoxy glue.

Table 2 Examples of 3 layers typical of SFA with their properties according to [8]

\begin{tabular}{lccc}
\hline layer & Thickness $[\mu \mathrm{m}]$ & $E[\mathrm{GPa}]$ & $v$ \\
\hline mica & 5.5 & 62 & 0.21 \\
epoxy & 25 & 3.4 & 0.5 \\
silica & substrate & 72 & 0.25 \\
\hline
\end{tabular}

However, notice once again that all the results are typically plotted in terms of contact area vs. load, which does not permit us to distinguish between force or displacement control. A realistic setup, of course, is somewhere in between load control and displacement control, the latter being the limit when the stiffness of the system tends to very high values.

In this paper, we shall extend the method published by Constantinescu et al. [9] to adhesive configurations and study some implications for SFA apparatuses JKR adhesive curves.

\section{FROM NON-ADHESIVE TO ADHESIVE SOLUTION}

The original adhesionless method [9] writes the harmonic Papkovich-Neuber displacement potentials as the Hankel transform of four unknown arbitrary functions $A_{1}^{i}(\lambda), A_{2}^{i}(\lambda), A_{3}^{i}(\lambda), A_{4}^{i}(\lambda)$ for each layer and two other functions $A_{5}(\lambda)$ and $A_{6}(\lambda)$ for the substrate. In order to get the solution of the problem, the determination of $4 n+2$ unknown functions is needed. Then we write the boundary conditions of continuity of displacements and traction among the layers, and finally the contact problem at the surface. The algebraic computation of displacements and stresses is done symbolically in Mathematica. The system has a solution in terms of $A_{1}^{1}(\lambda)$, which depends on an unknown pressure distribution (function $H(\tau)$ ). This function is the solution of the Fredholm equation of the second kind

$$
H(\tau)-\frac{1}{\pi} \int_{0}^{1} M(y, \tau) H(y) d y=F(\tau), \text { for } 0 \leq \tau \leq 1
$$

where $F$ depends only on the imposed indentation depth and on the indenter shape, and $\tau$ is the normalized radial coordinate in the contact area. For kernel $M(y, \tau)$, infinite integrals are given in Constantinescu et al. [9] which unfortunately contain highly oscillatory integrand if $a / h_{1}$ (the contact area over thickness of the first layer) is high. To improve the range of $a / h_{1}<100$ with sufficient accuracy, we modified the original code in the supplementary material of Constantinescu et al. [9], by splitting the integration intervals of the infinite integrals (which are obviously already truncated in practice to where the integrand is significantly nonzero) in 10 parts, where on each of them Gauss-Legendre quadrature with 25 points is done. 


\subsection{Transformation into adhesive solution}

The original JKR theory is derived and it determined the elastic strain energy $U$ for the sphere problem by following a two-step scenario as follows. We first load the contact in compression to load $P_{1}$ up to a contact area $A_{1}$. We then hold the contact area constant as to make the system linear, and then reduce the load to $P_{2}$. Hence we can write

$$
P_{2}=P_{1}-\left(\delta_{1}-\delta_{2}\right)\left(\frac{\partial P}{\partial \delta}\right)_{\delta_{1}}
$$

Assuming the final value of displacement $\delta_{2}$ [displacement control], contact areas $A_{1}$ and $\delta_{1}$ are obtained by minimizing the total potential energy

$$
\Pi=U-A_{1} \varpi
$$

Therefore, we obtain

and hence

$$
\frac{\partial \Pi}{\partial A_{1}}=0 \text { and hence } \frac{\partial U}{\partial A_{1}}=\frac{\partial U}{\partial \delta_{1}} \frac{\partial \delta_{1}}{\partial A_{1}}=\varpi
$$

$$
\delta_{2}=\delta_{1}-\sqrt{2 \varpi \frac{\partial A_{1}}{\partial \delta_{1}} / \frac{\partial^{2} P_{1}}{\partial \delta_{1}^{2}}}
$$

as obtained in [10], which permits us to derive a general relation between the adhesive solution and that without adhesion, which is exact in axisymmetric problems as is the present one. A similar derivation was later also suggested by Popov [11], and more restricted cases also treated in $[12,13]$.

We use non-dimensional parameters according to [1]:

$$
\hat{P}=\frac{P}{\pi R \varpi} ; \quad \hat{\delta}=\beta^{2} \frac{\delta}{R} ; \quad \hat{a}=\beta \frac{a}{R}
$$

where

$$
\beta=\left(\frac{E_{1}^{*} R}{\varpi}\right)^{1 / 3}
$$

with $E_{1}^{*}=\frac{E_{1}}{1-v_{1}^{2}}$ being the reduced elastic modulus of the first layer.

We obtain that pull-off under displacement control is $\hat{P}_{A}=-5 / 6 \pi$ and under force control it is $\hat{P}_{A}=-1.5 \pi$ for the original JKR spherical case. 


\section{SOME EXAMPLES}

Let us gives a few examples of interest for SFA apparatuses. In particular we consider a rigid sphere of radius $R=4 h_{1}$ indenting a layered flat surface. We take, therefore, as in Fig.1, 2 layers on a substrate having the same Young's modulus of to the top layer, with $E_{1}$ $=E_{3}=70 \mathrm{GPa}, v_{1}=0.21, v_{2}=0.5, v_{3}=0.25$ and we fix $E_{1} / E_{2}=20$ and $h=1 \mathrm{~nm}$, varying the thickness of intermediate layer $h_{2} / h_{1}=\{1,5,10,20,50\}$.

The solution is calculated over a list of adhesiveless indentation depth values. We use two discretizations: in the interval $\delta_{1}=[0.001,2] \mathrm{nm}$ we use a step of $0.02 \mathrm{~nm}$, while for $\delta_{1}=[2,50]$ we use a step of $0.5 \mathrm{~nm}$. The finer discretization for small values of $\delta_{1}$ aims at obtaining a good estimation of the pull-off in displacement control. It should be noted that it is not possible to compute the solution at point $\delta_{1}=0$, because of numerical limitation in the code.

As shown in Fig.2, the curves of load-indentation displacement or contact radius vs. displacement vary considerably their shape, and in particular the pull-off load vary both if under displacement control (the load at the lowest displacement, $\hat{P}_{A}$ ) or under forcecontrol $\hat{P}_{B}$ (the absolute minimum of the load).
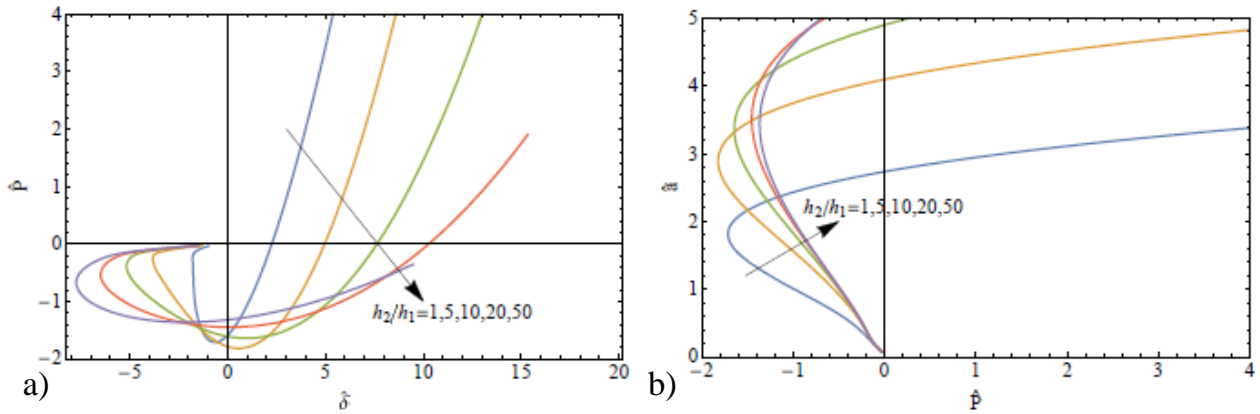

Fig. 2 Curves of load vs. displacement (a) and contact radius vs. load (b), for a case "SFA-like" (2 layers on a substrate having properties identical to the top layer), with $E_{1}=70 \mathrm{GPa} ; E_{1} / E_{2}=20$; but varying the thickness of the intermediate layer $h_{2} / h_{1}=$ $\{1,5,10,20,50\}$
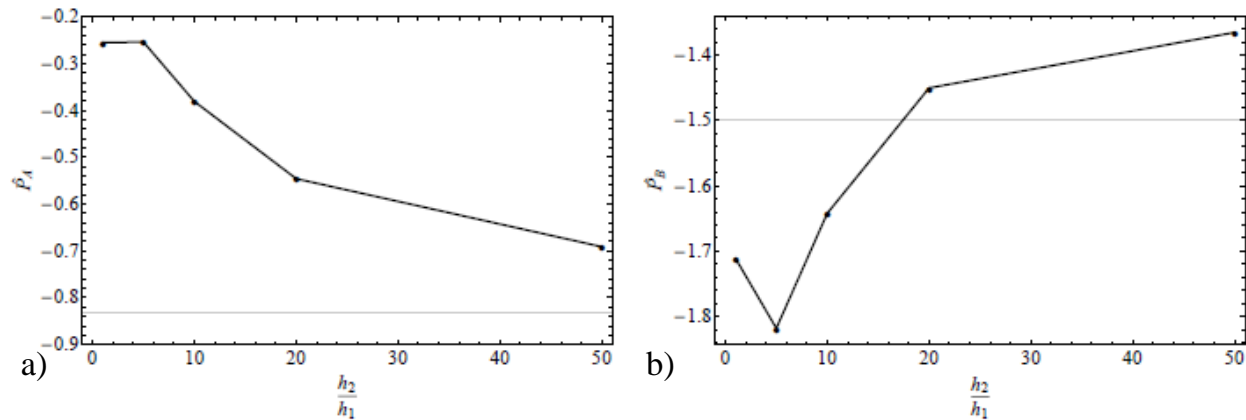

Fig. 3 Pull-off load under displacement-control $\hat{P}_{A}$ (a) or load control $\hat{P}_{B}$ (b) for a case "SFA-like" (2 layers on a substrate having properties identical to the top layer), with $E_{1}$ $=70 \mathrm{GPa} ; E_{1} / E_{2}=20$; but varying the thickness of the intermediate layer $h_{2} / h_{1}$ 
In particular, Fig. 3 shows only the pull-off loads and it is clear that the variation is particularly relevant under displacement control, which is a case not so documented in the literature, whereas under force control the variation is relatively small, as already wellknown.

We now move to consider a fixed ratio of thicknesses, namely $h_{2} / h_{1}=5$, which is typical for SFA, and vary modulus ratio $E_{1} / E_{2}=\{1,5,10,20,50\}$, see Figs. 4 and 5 . The variation of pull-off forces is shown to depend strongly on the modulus ratio initially and then asymptotically reach some limit values.
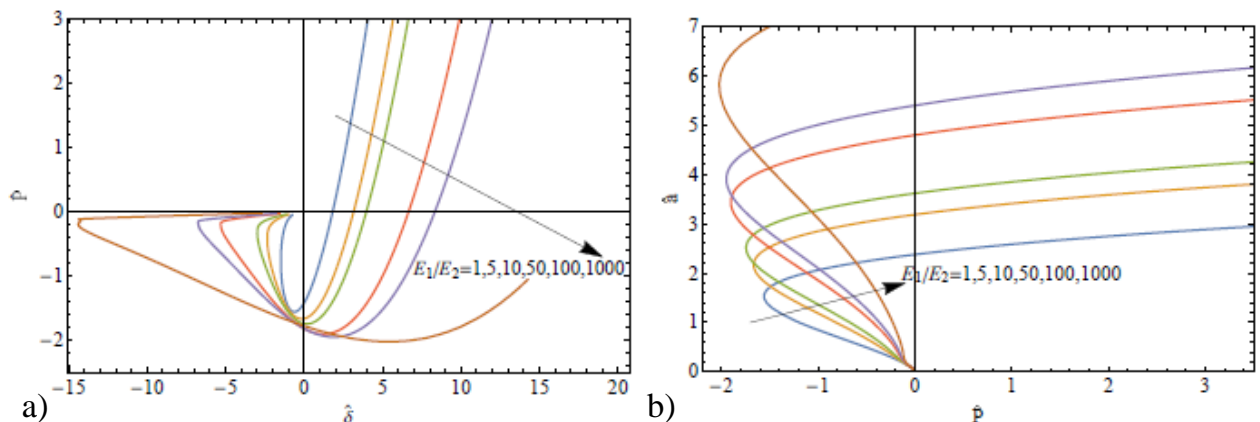

Fig. 4 Curves of load vs. displacement (a) and contact radius vs. load (b), for a case "SFA-like" with $h_{2} / h_{1}=5$, and vary the modulus ratio $E_{1} / E_{2}=\{1,5,10,20,50\}$
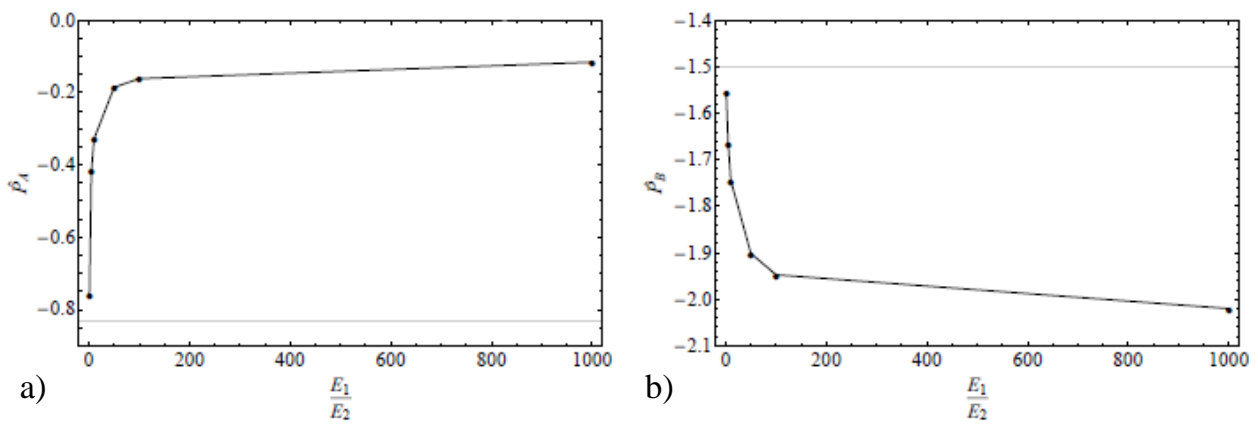

Fig. 5 Pull-off load under displacement-control $\hat{P}_{A}$ (a) or load control $\hat{P}_{B}$ (b) for a case "SFA-like" with $h_{2} / h_{1}=5$ and vary the modulus ratio $E_{1} / E_{2}=\{1,5,10,20,50\}$

\section{CONCLUSIONS}

We have obtained a general method for solving multilayered problems indentation with JKR adhesion and we have made some considerations for the SFA type of geometry. It is shown that the thickness ratio and modulus ratio of the second to first layers (assuming the substrate is almost identical to the top layer) can vary in a relatively modest way the pull-off under force control, and in a more pronounced way the pull-off under displacement control. The curves vary their shape considerably. The model provides a fast evaluation of the entire curve. 


\section{APPENDIX: MATHEMATICA CODE}

The code used for this work, implemented in the symbolic software Mathematica, is available as supplementary resource with the paper [13] at the following link: http://dx.doi.org/10.1016/j.ijsolstr.2013.04.017

Acknowledgements: Antonio Papangelo is thankful to the DFG (German Research Foundation) for funding the projects HO 3852/11-1 and PA 3303/1-1.

\section{REFERENCES}

1. Ciavarella, M., Joe, J., Papangelo, A., Barber, J.R., 2019, The role of adhesion in contact mechanics, Journal of the Royal Society Interface, 16(151), 20180738.

2. Johnson, K.L, Kendall, K., Roberts, A.D., 1971, Surface energy and the contact of elastic solids, Proceedings of the Royal Society A, 324(1558), pp. 301-313.

3. Tabor, D., Winterton, R.H.S, 1969, The Direct Measurement of Normal and Retarded van der Waals Forces, Proceedings of the Royal Society A, 312(1511), pp. 435-450.

4. Israelachvili, J.N., Tabor, D., 1972, The Measurement of Van Der Waals Dispersion Forces in the Range 1.5 to $130 \mathrm{~nm}$, Proceedings of the Royal Society A, 331(1584), pp. 19-38.

5. Sridhar, I., Johnson, K.L., Fleck, N.A., 1997, Adhesion mechanics of the surface force apparatus, Journal of Physics D: Applied Physics, 30(12), 1710.

6. Chen, S., Yan, C., Zhang, P., Gao, H., 2009, Mechanics of adhesive contact on a power-law graded elastic halfspace, Journal of the Mechanics and Physics of Solids, 57(9), pp. 1437-1448.

7. Stan, G., Adams, G.G., 2016, Adhesive contact between a rigid spherical indenter and an elastic multi-layer coated substrate, International Journal of Solids and Structures 87, pp. 1-10.

8. McGuiggan, P.M., Wallace, J.S., Smith, D.T., Sridhar, I., Zheng, Z.W., Johnson, K.L., 2007, Contact mechanics of layered elastic materials: experiment and theory, Journal of Physics D: Applied Physics, 40(19), 5984.

9. Constantinescu, A., Korsunsky, A.M., Pison, O., Oueslati, A., 2013, Symbolic and numerical solution of the axisymmetric indentation problem for a multilayered elastic coating, International Journal of Solids and Structures, 50(18), pp. 2798-2807.

10. Ciavarella, M., 2018, An approximate JKR solution for a general contact, including rough contacts, Journal of the Mechanics and Physics of Solids, 114, pp.209-218

11. Popov, V.L, 2018, Solution of adhesive contact problem on the basis of the known solution for non-adhesive one, Facta Universitatis-Series Mechanical Engineering, 16 (1), pp. 93-98.

12. Argatov, I., Li, Q., Pohrt, R., Popov, V.L., 2016, Johnson-Kendall-Roberts adhesive contact for a toroidal indenter, Proceedings of the Royal Society A, 472(2191), 20160218.

13. Popov, V.L., Hess, M., Willert, E., 2017, Handbuch der Kontaktmechanik: Exakte Lösungen axialsymmetrischer Kontaktprobleme, Springer, Berlin, 341 p. 